Research Article

\title{
Raceway Defect Frequency Deviation of Full-Ceramic Ball Bearing Induced by Fit Clearance in Wide Temperature Ranges
}

\author{
Xiaotian Bai $\mathbb{D},{ }^{1}$ Hao Zheng $\mathbb{D},{ }^{1}$ Zinan Wang $\mathbb{D},{ }^{1}$ and Zhong Wang $\mathbb{D}^{2}$ \\ ${ }^{1}$ School of Mechanical Engineering, Shenyang Jianzhu University, Shenyang, Liaoning 110168, China \\ ${ }^{2}$ School of Mechanical Engineering, Liaoning Institute of Science and Technology, Benxi, Liaoning 117004, China \\ Correspondence should be addressed to Zinan Wang; wzn1404589743@126.com
}

Received 22 December 2020; Revised 10 January 2021; Accepted 17 March 2021; Published 20 April 2021

Academic Editor: Rozaimi Ghazali

Copyright (c) 2021 Xiaotian Bai et al. This is an open access article distributed under the Creative Commons Attribution License, which permits unrestricted use, distribution, and reproduction in any medium, provided the original work is properly cited.

Full-ceramic ball bearings are widely applied in wide temperature ranges due to their excellent thermal shock resistance, and the condition monitoring and fault diagnosis are mainly conducted through the spectrum analysis based on the defect frequencies. However, the outer ring has a spinning motion in the temperature-related fit clearance, which leads to the deviation of raceway defect frequencies, and is not conducive to the fault diagnosis. In this paper, the temperature-related fit clearance is considered in the dynamic model, and defects are added on the inner raceway and outer raceway. The motions of the rings are calculated and analyzed in the frequency domain, and the trends of peak frequencies with temperature are investigated. Simulation and experimental results show that the spinning speed of the outer ring increases with temperature, and the defect frequencies exhibit obvious deviation in wide temperature ranges. In a temperature range of $500 \mathrm{~K}$, the defect frequencies exhibit deviations of over $3 \%$, which is obvious in the defect frequency identification. The results provide insights on the full-ceramic ball bearing dynamics and help with the fault diagnosis and status monitoring of the relevant devices.

\section{Introduction}

Rolling bearings are fundamental elements in rotary machines, whose operation stability is significant for the whole mechanical system. In many advanced areas such as aerospace and nuclear industries, rolling bearing systems often work in wide temperature ranges, thus providing higher requirements for the thermal shock resistance of rolling bearings. Full-ceramic ball bearings exhibit more stability in wide temperature ranges due to the small thermal deformation and are, therefore, more preferred over steel bearings in working conditions with wide temperature ranges $[1,2]$. The balls and rings of full-ceramic ball bearings are made of engineering ceramic materials such as silicon nitride and zirconia, thus providing high stiffness and wear resistance to the bearing system. In the continuous operation of ceramic bearing systems, defects such as cracks and spallings often appear on the inner and outer raceways under impacts. The ceramic bearing components have poor resistance to surface cracking and spalling as a result of the unavailability of plastic binding phases, which results in the sharp degrading in performance when defects occur. Therefore, the condition monitoring and fault diagnosis for ceramic bearings are extremely important for the stable operation of related devices. Recently, the fault diagnosis is mainly conducted in the frequency domain, and the peak frequencies of the bearing system are compared with the preobtained defect frequencies for fault identification. For full-ceramic bearing systems, the outer ring becomes loose due to the temperature-related fit clearance. The motion of the outer ring leads to nonlinear interactions between bearing components, and the defect frequencies also vary obviously, which need to be studied in detail.

Setting up dynamic models is one of the main approaches to the interactions between bearing components, and many studies have been carried out in the recent years [3-7]. Xi et al. [8] presented a dynamic model of the spindle bearing system with ball bearing and spindle housing and carried out investigations on the bearing performance in different working conditions. Bizarre et al. [9] formulated a 
ball bearing model with five degrees of freedom accounting for the effects of the elastohydrodynamic effect and suggested that the contact between bearing elements was affected by axial and moment loads and lubrication conditions. Li et al. [10] established a mechanical model of ball bearing with a localized defect on the outer raceway and analyzed the change of contact angles and load distributions with varying rotation speeds. Yan et al. [11] considered the skidding effect between balls and rings in the fault dynamic model and applied the impulse envelope signal phase in the fault signature enhancement to eliminate the order deviation and vagueness phenomenon. Molano et al. [12] built a bearing dynamic model with localized faults on the inner ring and outer ring for the Independent Cart Conveyor System and considered the modulation of load on the bearings and random contributions to get the fault features in the frequency domain. Yu et al. [13] took the time-varying effect caused by the distributed and localized faults into consideration and found that the faults not only influenced the system on the dynamic behaviors but also on the geometric topographies. It is generally accepted that the surface defects affect the interactions between bearing components by changing the contact forces, and the defect frequencies are obtained through the relative motions between rings and balls [14-16]. However, for full-ceramic ball bearings, the fit clearance between the outer ring and the pedestal changes greatly in wide temperature ranges. The relative motions between the rings need to be considered to ensure the defect frequencies.

The fit clearance exists widely in bearing systems and causes nonlinear dynamic behaviors inevitably [17]. Chen and $\mathrm{Qu}$ [18] studied the effect of fit clearance on the vibration response and pointed out that the fit clearance led to the periodic contact between the outer ring and the pedestal. Cao et al. [19] considered the effect of fit clearance in the bearing-rotor-pedestal coupled model and found that multiple harmonics appeared in the response due to the nonlinear self-oscillation caused by the fit clearance. Mao et al. [20] proposed a model with the ring flexibility and pedestal contact force, and the results showed that the fit clearance made an impact on the system vibration by changing the load distribution. It has been proved in previous studies $[21,22]$ that the fit clearance for full-ceramic bearing had a nonlinear growth with the working temperature, and the outer ring made an orbit-spinning compound motion in the pedestal with the effect of friction torque. As a result, it can be predicted that changes will occur to the relative velocities between the balls and rings. The change of relative velocities directly leads to the deviation of defect frequencies and brings difficulties to the fault detection of full-ceramic bearing systems at different temperatures.

Therefore, a precise dynamic model with faults and the effect of temperature-related fit clearance is quite essential. In this study, the interactions with the effect of fit clearance are analyzed, and the relative motions between balls and rings are obtained for the defect frequency deviation. The model is presented in Section 2. The impacts of fit clearance on the outer ring motions are analyzed in Section 3. In Section 4, the calculation values are compared with the experimental results for verification. The trends of the motion with working conditions are discussed in Section 5. Conclusions are presented in Section 6.

\section{Fault Dynamic Model with Fit Clearance}

The full-ceramic bearings are usually installed in steel pedestals. For the convenience of assembling, the fits between the outer ring and the pedestal are usually set as clearances, leaving a positive clearance to make it easier to adjust the axial position of the bearing. However, when the working temperature changes, the difference in thermal deformation between the outer ring and the pedestal leads to the variance in fit clearance. Assuming that the thermal deformation of the components is uniform in all directions, the deformation anistropy of the ceramic material and the boundary effect that occurred at the edges are ignored. The bore diameter of the outer ring and the outline diameter grow synchronously, and the deformation is shown in Figure 1.

In Figure 1, the dashed lines show the outlines at the initial temperature $T_{0}$, while the solid lines show the outlines after deformation. Here, $D_{1}$ shows the initial diameter of the pedestal, and $L$ is the initial pedestal width. $D_{0}$ is the initial bore diameter of the pedestal, and $d_{0}$ is the initial diameter of the outer ring. The initial fit clearance $\delta_{0}$ can be expressed as

$$
\delta_{0}=D_{0}-d_{0} .
$$

And, the fit clearance after thermal deformation can be expressed as

$$
\delta_{0}^{\prime}=\sqrt{\frac{D_{1}^{2}\left(1+\lambda_{\mathrm{p}} \Delta T\right)^{3}-\left(D_{1}^{2}-D_{0}^{2}\right)\left(1+3 \lambda_{\mathrm{p}} \Delta T\right)}{1+\lambda_{\mathrm{p}} \Delta T}}-d_{0} \cdot\left(1+\lambda_{\mathrm{o}} \cdot \Delta T\right),
$$

where $\delta_{0}^{\prime}$ is the fit clearance after deformation, $\lambda_{\mathrm{p}}$ is the thermal deformation coefficient of the pedestal, $\lambda_{\mathrm{o}}$ is the thermal deformation coefficient of the outer ring, and $\Delta T$ is the temperature range and can be expressed as

$$
\Delta T=T-T_{0},
$$

where $T$ is the working temperature. Then, the contact model with clearance is shown in Figure 2.

In Figure 2, the coordinate system $\{O ; X, Y, Z\}$ is set as the reference system, in which $O$ is the center of the pedestal bore, $O X$ is the axial direction, and $O Y$ and $O Z$ are radial directions. The coordinate system $\left\{O_{i} ; X_{i}, Y_{i}, Z_{i}\right\}$ shows the position of the inner ring, and $\left\{O_{o} ; X_{o}, Y_{o}, Z_{o}\right\}$ shows the position of the outer ring. $O_{i}$ is set at the center of the inner ring, and $O_{o}$ is set at the center of the outer ring. The balls are driven by the inner ring and slide along the outer ring raceway. $d_{i}$ is the diameter of the inner raceway, $d_{m}$ is the pitch diameter, and $d_{o}$ is the diameter of the outer raceway. Here, the cage is not shown for clarity. As the thermal deformation of the ceramic component is relatively small, the thermal deformation of the components is regarded as free expansion, which is the same as the outer ring. Only the radial motions of the bearing system are discussed in this 


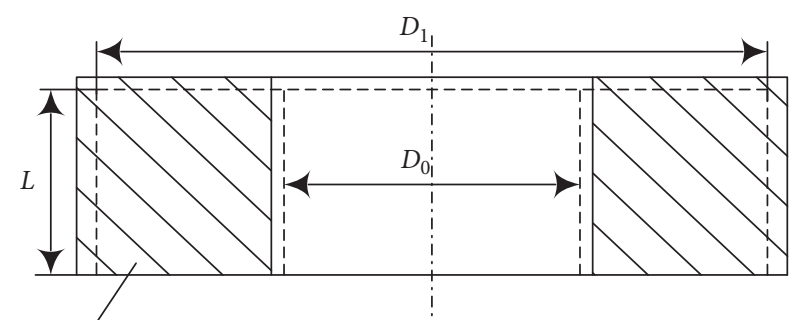

Pedestal

(a)

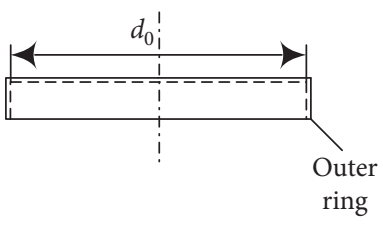

(b)

Figure 1: Thermal deformation. (a) The pedestal. (b) The outer ring.

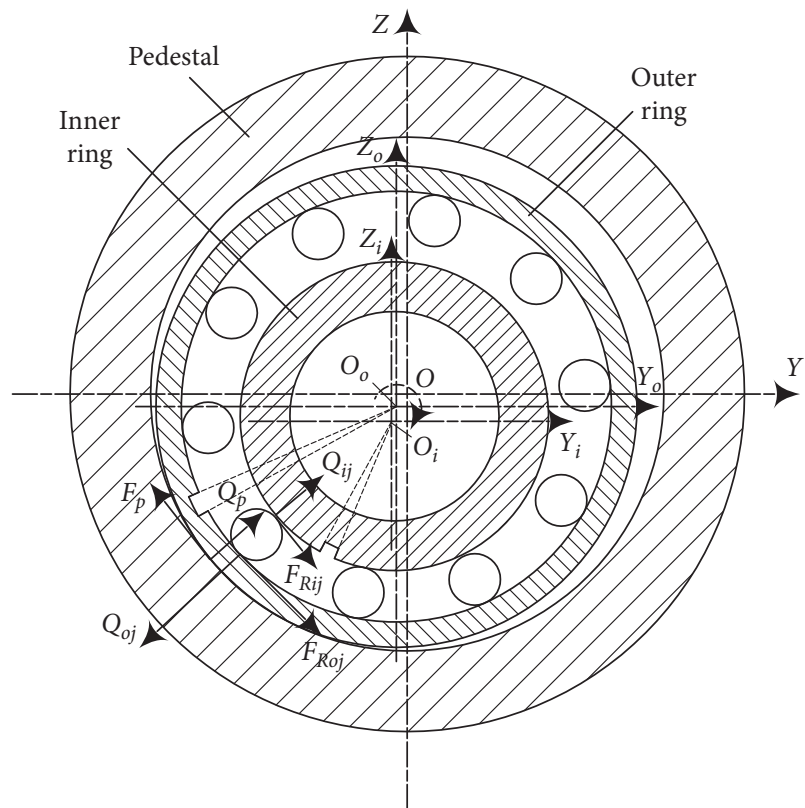

(a)

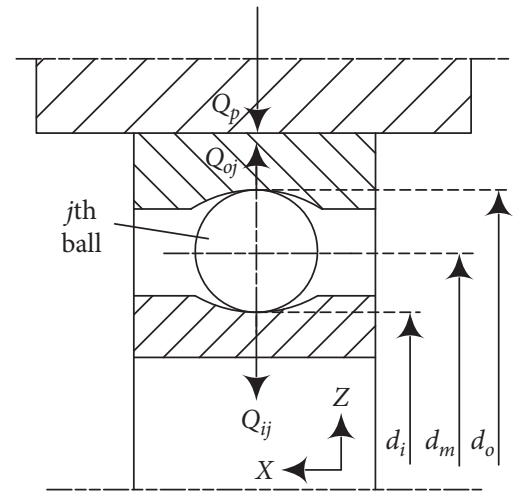

(b)

FIGURE 2: Contact model with clearance in various planes. (a) YOZ plane. (b) XOZ plane.

paper, so the movements around $O Y$ and $O Z$ are ignored. When the balls are running on the regions without defects, the motion of the inner ring can be described as

$$
\begin{array}{r}
F_{e} \cos \phi_{i}-\sum_{j=1}^{N}\left(Q_{i j} \cos \psi_{j}+F_{R i j} \sin \psi_{j}\right)=m_{i} \ddot{y}_{i}, \\
F_{e} \sin \phi_{i}-\sum_{j=1}^{N}\left(Q_{i j} \sin \psi_{j}-F_{R i j} \cos \psi_{j}\right)-m_{i} g=m_{i} \ddot{z}_{i},
\end{array}
$$

where $F_{e}$ shows the centrifugal force, $\phi_{i}$ is the eccentric angle in $\{O ; Y, Z\}, Q_{i j}$ is the contact force acting on the inner ring, $F_{R \mathrm{ij}}$ is the friction force between the inner ring and the $j$ th ball in the $Y O Z$ plane, $\psi_{j}$ is the azimuth angle of the $j$ th ball in $\left\{O_{i} ; Y_{i}, Z_{i}\right\}, m_{i}$ is the mass of the inner ring, $N$ is the total number of balls, and $x_{i}$ and $y_{i}$ are displacements of the inner ring, respectively. Here, the mass and force of the rotor are transferred and lumped on the inner ring, and the motion of the outer ring is shown as

$$
\begin{gathered}
\sum_{j=1}^{N}\left(Q_{o j} \cos \phi_{j}-F_{R o j} \sin \phi_{j}\right)-Q_{p} \cos \phi_{o}+F_{p} \sin \phi_{o}=m_{o} \ddot{y}_{o}, \\
\sum_{j=1}^{N}\left(Q_{o j} \sin \phi_{j}+F_{R o j} \cos \phi_{j}\right)-Q_{p} \sin \phi_{o}-F_{p} \cos \phi_{o}-m_{o} g=m_{o} \ddot{z}_{o},
\end{gathered}
$$




$$
\sum_{j=1}^{N}\left[\frac{F_{R o j} \cdot\left(d_{m}+d_{b}\right)\left(1+\lambda_{o} \cdot \Delta T\right)}{2}\right]-F_{p} \cdot \frac{d_{0}\left(1+\lambda_{o} \cdot \Delta T\right)}{2}=J_{o} \cdot \dot{\omega}_{o}
$$

where $Q_{o j}$ is the contact force acting on the outer ring, $F_{R o j}$ is the friction force in the YOZ plane, $m_{o}$ is the mass of the outer ring, $J_{o}$ is the rotary inertia, $y_{o}$ and $z_{o}$ are displacements of the outer ring along $O Y$ and $O Z$ directions, $\omega_{o}$ is the rotation speed around $O_{o}, d_{b}$ is the ball diameter, $Q_{p}$ is the contact force from the pedestal, $F_{p}$ is the friction force on the outer ring, $\phi_{j}$ is the azimuth angle in $\left\{O_{o} ; Y_{o}, Z_{o}\right\}$, and $\phi_{\mathrm{o}}$ is the eccentric angle of the outer ring in $\{O ; Y, Z\}$. For the azimuth and eccentric angles, $O Y$ is set as the reference direction, as shown in Figure 3.

In Figure 3, $e_{o}$ is the distance between $O$ and $O_{o}$ and can be expressed as

$$
e_{o}=\sqrt{y_{o}^{2}+z_{o}^{2}}
$$

where $e_{i}$ is the distance between $O$ and $O_{i}$ and can be expressed as

$$
e_{i}=\sqrt{y_{i}^{2}+z_{i}^{2}}
$$

The azimuth of $\psi_{j}$ can be considered to change uniformly as the balls are driven by the inner ring, and $\phi_{j}$ is related with $\psi_{j}$ as

$$
\left\{\begin{array}{l}
\phi_{j}=\psi_{j}+\Delta \phi, \quad \text { when } \quad \arctan \frac{z_{o}-z_{i}}{y_{o}-y_{i}}<\psi_{j}<\pi+\arctan \frac{z_{o}-z_{i}}{y_{o}-y_{i}}, \\
\phi_{j}=\psi_{j}-\Delta \phi, \quad \text { when } \quad \psi_{j}<\arctan \frac{z_{o}-z_{i}}{y_{o}-y_{i}}, \quad \text { or } \quad \pi+\arctan \frac{z_{o}-z_{i}}{y_{o}-y_{i}}<\psi_{j}<2 \pi+\arctan \frac{z_{o}-z_{i}}{y_{o}-y_{i}},
\end{array}\right.
$$

where $\Delta \phi$ can be obtained through the cosine theorem as

$$
\cos \Delta \phi=\frac{\left[\left(d_{i}+d_{m}\right)^{2}+\left(d_{o}-d_{m}\right)^{2}\right]\left(1+\lambda_{o} \cdot \Delta T\right)^{2}-4\left(y_{o}-y_{i}\right)^{2}-4\left(z_{o}-z_{i}\right)^{2}}{2\left(d_{i}+d_{m}\right)\left(d_{o}-d_{m}\right)\left(1+\lambda_{o} \cdot \Delta T\right)^{2}}
$$

When defects occur on the raceways, the balls pass through the defect area in sequence and bring about impacts due to the interrupted contacts, as shown in Figure 4.

In Figure $4, \psi_{s i}$ shows the azimuth of the defect on the inner raceway, $\phi_{\text {so }}$ shows the azimuth of the defect on the outer raceway, and the sizes of the defects are denoted by $\theta_{s i}$ and $\theta_{s o}$. For cases with a defect on the inner raceway, when the $j$ th ball enters the defect area, it loses contact with the inner raceway at first and then has an impact with the raceway at $\psi_{j}=\psi_{s i} . F_{i j}$ is the contact force acting on the inner ring when $\psi_{j}=\psi_{s i}$ and only counts when $\psi_{j}=\psi_{s i}$. $\theta_{i}$ shows the angle between $F_{i j}$ and $\psi_{s i}$, which can be obtained through

$$
\sin \theta_{i}=\frac{d_{i}}{d_{b}} \cdot \sin \frac{\theta_{s i}}{2} .
$$

Assuming that the motions of the balls on the raceways are pure rolling, the orbit speed of the balls can be expressed as

$$
\omega_{m}=\frac{\omega_{i} d_{i}+\omega_{o} d_{o}}{2 d_{m}}\left(1+\alpha_{o} \cdot \Delta T\right)
$$

And, $F_{i j}$ can be expressed as

$$
F_{i j}=\frac{m_{i}\left(\omega_{i}-\omega_{m}\right)^{2} d_{i} \cos \left(\theta_{s i} / 2\right)}{2 \cos \theta_{i}}\left(1+\alpha_{o} \cdot \Delta T\right) .
$$

Therefore, when an impact occurs on the inner raceway, equations (4) and (5) should be revised as

$$
F_{e} \cos \phi_{i}-\sum_{j=1}^{N}\left[Q_{i j} \cos \psi_{j}+F_{R i j} \sin \psi_{j}+F_{i j} \cos \left(\psi_{s i}-\theta_{i}\right)\right]=m_{i} \ddot{y}_{i},
$$

$$
\begin{aligned}
F_{e} \sin \phi_{i} & -\sum_{j=1}^{N}\left[Q_{i j} \sin \psi_{j}-F_{R i j} \cos \psi_{j}+F_{i j} \sin \left(\psi_{s i}-\theta_{i}\right)\right] . \\
& -m_{i} g=m_{i} \ddot{z}_{i}
\end{aligned}
$$

For cases with a defect on the outer raceway, $F_{o j}$ is the impact force when $\phi_{j}=\phi_{s o}$ and only counts when $\phi_{j}=\phi_{s o} . \theta_{o}$ is the angle between $F_{o j}$ and $\phi_{s o}$, which can be obtained through

$$
\sin \theta_{o}=\frac{d_{o}}{d_{b}} \cdot \sin \frac{\theta_{s o}}{2} .
$$

And, $F_{o j}$ can be expressed as 


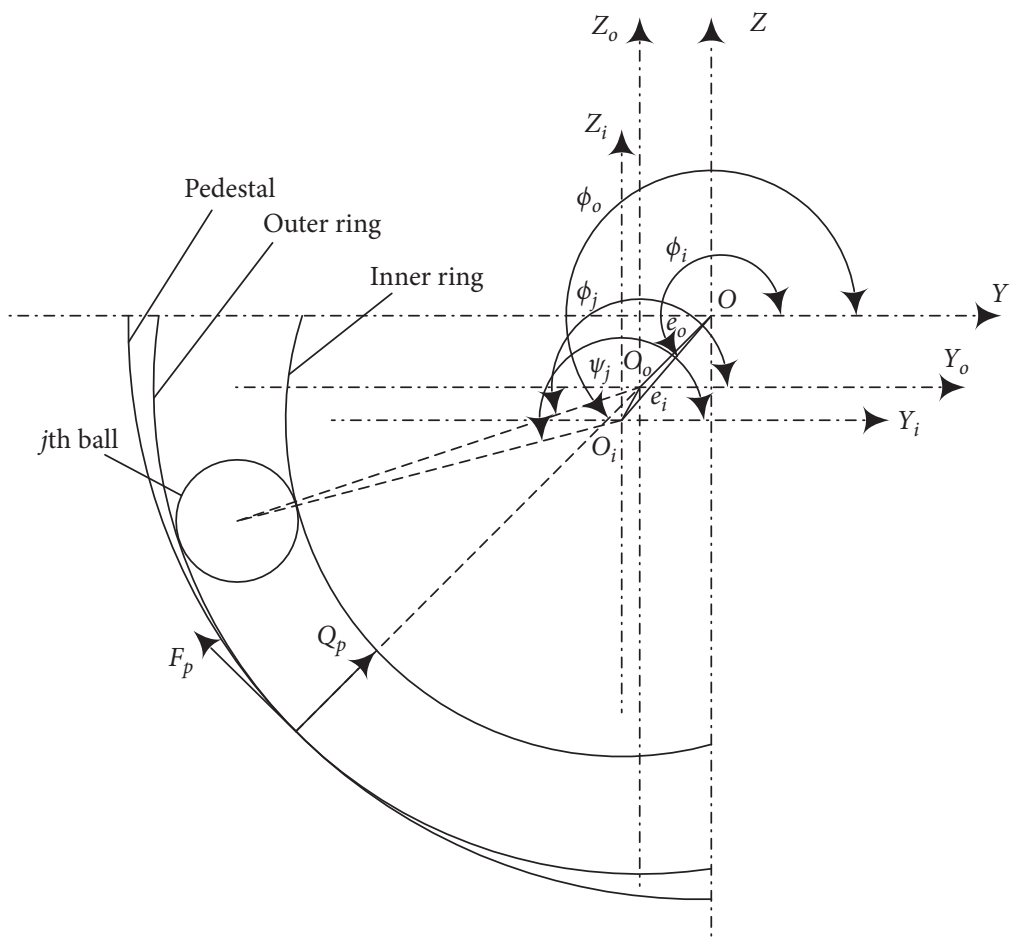

FIgURE 3: Contact between the outer ring and the pedestal.

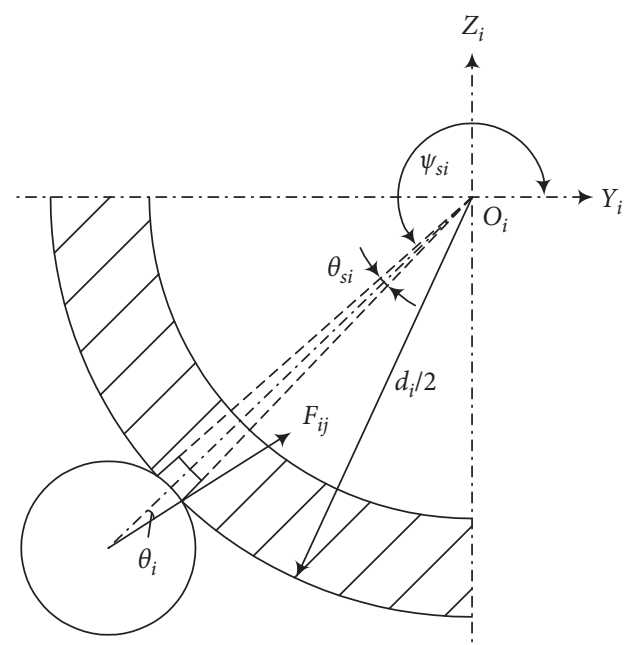

(a)

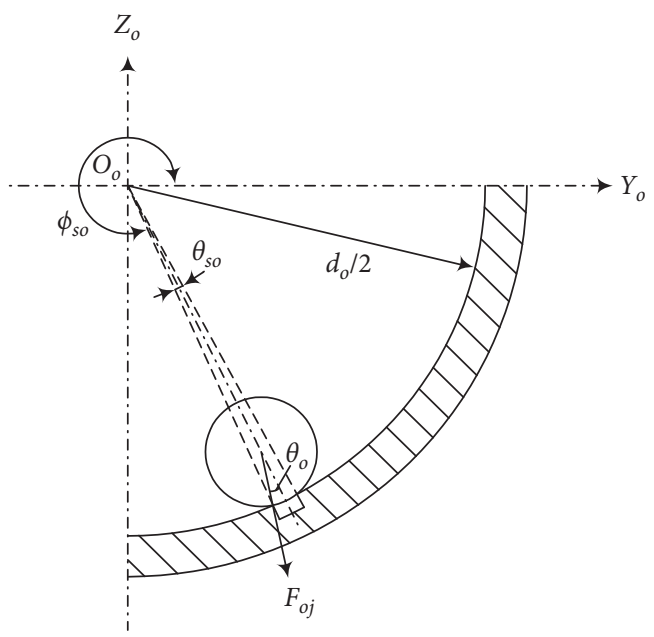

(b)

Figure 4: Contact between the ball and the ring when the defect occurs on different raceways. (a) On the inner raceway. (b) On the outer raceway. 


$$
F_{o j}=\frac{m_{j}\left(\omega_{m}-\omega_{o}\right)^{2}\left(d_{o} \cos \left(\theta_{s o} / 2\right)-d_{b} \cos \theta_{o}\right)}{2 \cos \theta_{o}}\left(1+\lambda_{o} \cdot \Delta T\right) .
$$

Then, when an impact occurs on the outer raceway, equations (6)-(8) should be revised as

$$
\begin{gathered}
\sum_{j=1}^{N}\left[Q_{o j} \cos \varphi_{j}-F_{R o j} \sin \varphi_{j}+F_{o j} \cos \left(\varphi_{s o}-\theta_{o}\right)\right]-Q_{p} \cos \varphi_{o}+F_{p} \sin \varphi_{o}=m_{o} \ddot{y}_{o} \\
\sum_{j=1}^{N}\left[Q_{o j} \sin \varphi_{j}+F_{R o j} \cos \varphi_{j}+F_{o j} \sin \left(\varphi_{s o}-\theta_{o}\right)\right]-Q_{p} \sin \varphi_{o}-F_{p} \cos \varphi_{o}-m_{o} g=m_{o} \ddot{z}_{o} \\
\sum_{j=1}^{N}\left[\frac{F_{R o j} \cdot d_{o}\left(1+\lambda_{o} \cdot \Delta T\right)}{2}-\frac{m_{j}\left(\omega_{m}-\omega_{o}\right)^{2}\left(d_{o} \cos \left(\theta_{s o} / 2\right)-d_{b} \cos \theta_{o}\right) \cdot \tan \theta_{o} d_{o}\left(1+\lambda_{o} \cdot \Delta T\right)^{2}}{4}\right]-\frac{F_{p} d_{o}\left(1+\lambda_{o} \cdot \Delta T\right)}{2}=J_{o} \cdot \dot{\omega}_{o}
\end{gathered}
$$

$F_{p}$ and $Q_{p}$ are taken into consideration only when the outer ring is in contact with the pedestal, that is,

$$
e_{\mathrm{o}} \geq \frac{\delta_{0}^{\prime}}{2}
$$

Assuming that the contact force submits Hooke's law within the small deformation, that is,

$$
Q_{\mathrm{p}}=k_{\mathrm{p}} \cdot\left(e_{\mathrm{o}}-\frac{\delta_{0}^{\prime}}{2}\right) \text {. }
$$

The friction force $F_{p}$ is related with $Q_{p}$ when the outer ring is in contact with the pedestal, and the direction of $F_{p}$ depends on the rotation of the outer ring, that is,

$$
F_{p}=f_{p} \cdot Q_{p} \cdot \frac{\dot{z}_{o} \cos \phi_{o}-\dot{y}_{o} \sin \phi_{o}+\omega_{o} \cdot\left(d_{0} \cdot\left(1+\lambda_{o} \cdot \Delta T\right) / 2\right)}{\left|\dot{z}_{o} \cos \phi_{o}-\dot{y}_{o} \sin \phi_{o}+\omega_{o} \cdot\left(d_{0} \cdot\left(1+\lambda_{o} \cdot \Delta T\right) / 2\right)\right|},
$$

where $f_{p}$ is the friction coefficient between the outer ring and the pedestal. When $e_{0}<\delta_{0}^{\prime} / 2$, it can be considered that the outer ring separates from the pedestal, and $Q_{p}=F_{p}=0$. Then, it is obvious that, as the working temperature increases, the fit clearance becomes larger, and the outer ring spins in the pedestal bore with the effect of $F_{p}$. It can be inferred that $\omega_{o}$ has a complex variance in a wide temperature range due to the fit clearance. When $\omega_{o}$ changes, the impact frequency also changes, as shown in equations (14)-(21), and the effect of the fit clearance can be revealed in the dynamic response of the inner ring and outer ring.

\section{Numerical Simulation}

To test the performance of the full-ceramic bearing system, numerical simulations at different temperatures are essential. The thermal deformation of the bearing and pedestal are expressed in equations (1)-(3), and the clearance between the outer ring and pedestal can be used as the geometry boundary conditions; then, the motions of the bearing can be obtained through equations (16) and (17) and (20)-(22). Assuming that, at time $t=0$, the ball of $j=1$ is located at $\psi_{j}=0^{\circ}$, the outer ring is in contact with the pedestal at $\phi_{o}=0^{\circ}$, and the radial load is set as $100 \mathrm{~N}$. Then, the dynamic response of the inner ring and outer ring can be obtained through iterative calculation, and the flowchart is shown in Figure 5.

Here, the initial temperature is set as $T_{0}=100 \mathrm{~K}$, and the initial fit clearance $\delta_{0}$ is $0.003 \mathrm{~mm}$. The structural parameters of the full-ceramic ball bearing and the pedestal are listed in Table 1.

The pedestal is made of steel, and the balls and rings of the full-ceramic ball bearing are made of silicon nitride. The physical parameters of the bearing components are included in Table 2.

3.1. Dynamic Response with the Inner Raceway Defect in Different Temperatures. The maximum temperature range $\Delta T$ is set as $500 \mathrm{~K}$ in this study, and the working temperature $T$, which can be expressed as $T=T_{0}+\Delta T$, ranges from $100 \mathrm{~K}$ to $600 \mathrm{~K}$. The rotation speed of the inner ring is set as $2400 \mathrm{r} /$ $\mathrm{min}$ in the anticlockwise direction, and the radial load is set as $100 \mathrm{~N}$. It is assumed that the shapes of the raceways are ideal arcs, and the balls are ideal spheres with identical diameters, the effects of surface waviness and shape errors are ignored. The friction coefficient between the bearing components is 0.1 , and the friction coefficient between the outer ring and the pedestal is 0.2 . The external shock is not taken into consideration. At the initial time $t=0$, the defect is located at $\psi_{s i}=0^{\circ}$. The size of the defect is $\theta_{s i}=2^{\circ}$, and the balls are not in touch with the bottom of the defect. The temperature step is $100 \mathrm{~K}$, and the vertical speed $\dot{z}_{\mathrm{i}}$ is selected as the indicator. The time step for the calculation is $0.0004 \mathrm{~s}$, and the dynamic responses in different temperatures are shown in Figure 6.

It can be seen from Figure 6 that the velocity of the inner ring is approximate to sinusoidal variation, and the amplitude gradually increases with working temperature. The 


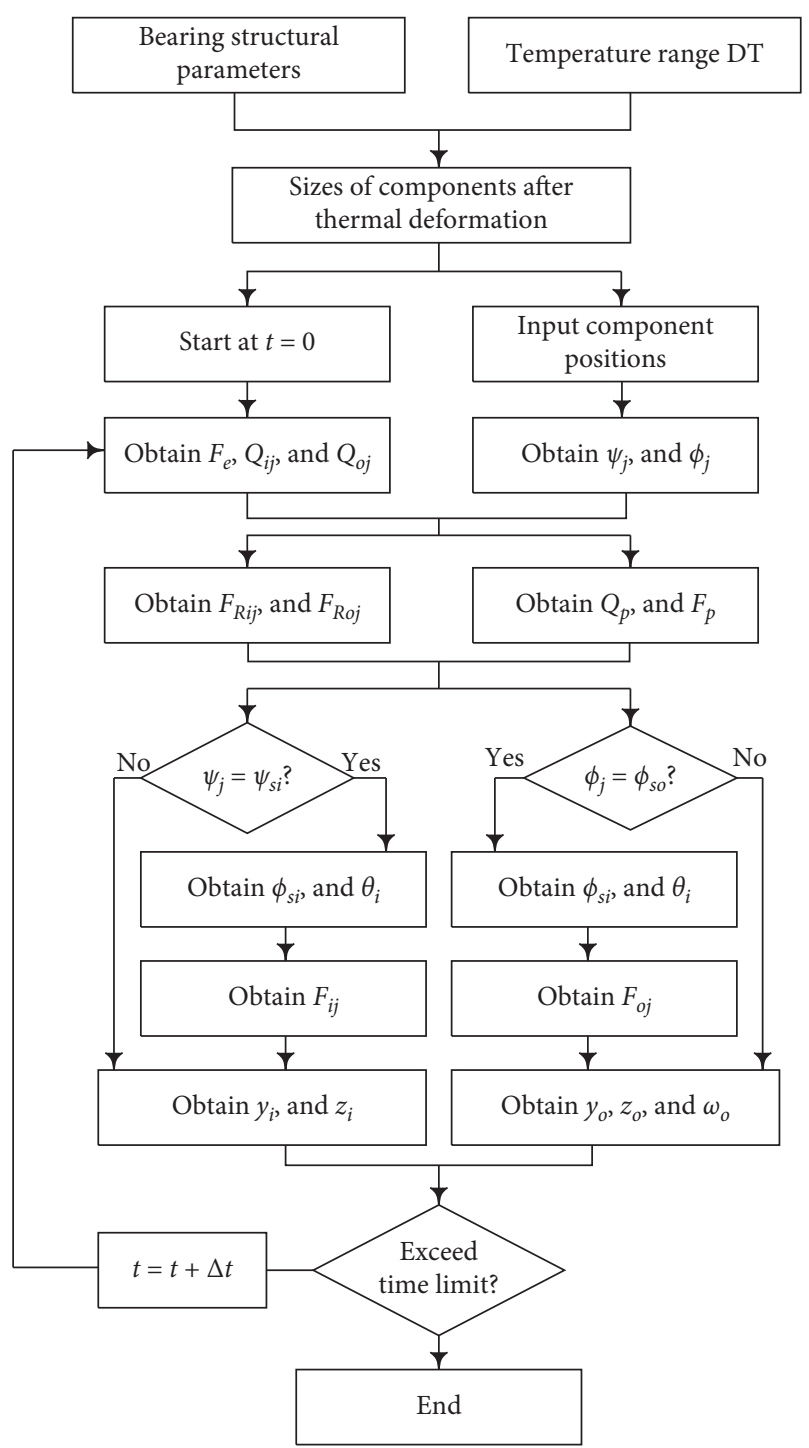

FIGURE 5: Calculation flowchart.

TABle 1: Parameters of ceramic ball bearings and the pedestal.

\begin{tabular}{lc}
\hline Item & Value \\
\hline Bearing pitch diameter (inch) & 1.318 \\
Contact angle (degree) & 0 \\
Bearing width (mm) & 15 \\
Ball diameter (inch) & 0.3125 \\
Number of balls & 8 \\
Pedestal diameter (mm) & 120 \\
Pedestal width (mm) & 10 \\
\hline
\end{tabular}

TABLe 2: Physical parameters of the bearing components.

\begin{tabular}{lc}
\hline Item & Value \\
\hline Bearing density $\left(\mathrm{kg} / \mathrm{m}^{3}\right)$ & 3100 \\
Pedestal density $\left(\mathrm{kg} / \mathrm{m}^{3}\right)$ & 7850 \\
Bearing elastic modulus $(\mathrm{Pa})$ & $3.3 \times 10^{11}$ \\
Pedestal elastic modulus $(\mathrm{Pa})$ & $2.16 \times 10^{11}$ \\
Thermal deformation coefficient of the pedestal $(1 / \mathrm{K})$ & $1.25 \times 10^{-5}$ \\
Thermal deformation coefficient of the bearing $(1 / \mathrm{K})$ & $2.8 \times 10^{-6}$ \\
Contact stiffness of the pedestal $(\mathrm{N} / \mathrm{m})$ & $2.5 \times 10^{8}$ \\
\hline
\end{tabular}

peak values appear periodically in the time domain, which is caused by the impacts on the defect area. To get the frequency components in the velocities, the results are processed by FFT with the analysis frequency of $2500 \mathrm{~Hz}$, as shown in Figure 7.

As indicated in Figure 7, the frequency components contained in the inner ring velocities are related with the rotating frequency and defect frequency. Obviously, the peaks at $40 \mathrm{~Hz}$ and $80 \mathrm{~Hz}$ correspond to the rotating frequency and doubling rotating frequency, and the peak near $200 \mathrm{~Hz}$ is the defect frequency. The defect frequency and amplitudes at each peak in different temperatures are given in Table 3.

The peak corresponding to the rotating frequency is the highest, which implies that the centrifugal force caused by the bearing clearance and fit clearance makes the most contribution to the inner ring vibration. The doubling rotating frequency can also be seen in the result, but the amplitude is much smaller. As the fit clearance increases with the working temperature, the maximum eccentricity grows, and the centrifugal force from the rotor and the inner ring increases to bring about the amplitude growth of the rotating frequency-related components. With the growth of the centrifugal force, the impact of balls crossing the defect area also becomes severer, and the amplitude of the defect frequency also increases with the temperature, as shown in Figure 7.

According to equation (22), the outer ring begins to rotate as the outer ring becomes loose with the increasing fit clearance. The outer ring has an opposite rotation direction to the inner ring, and the orbit speed of the balls decreases according to the pure rolling hypothesis. Then, the relative velocities between the inner ring and the balls increase, and the characteristic frequency for the defect on the inner raceway turns larger, which has the consistent trend as Table 3.

3.2. Dynamic Response with the Outer Raceway Defect in Different Temperatures. The working condition parameters are set the same as in the previous section, and the temperature also ranges from $100 \mathrm{~K}$ to $600 \mathrm{~K}$. At the initial time $t=0$, the defect is set at $\phi_{s o}=270^{\circ}$, and the defect has an opening angle of $\theta_{s o}=2^{\circ}$. The balls never touch the bottom of the defect during rotation. The time step is $0.0004 \mathrm{~s}$; then, the vertical speed of the outer ring $\dot{z}_{\mathrm{o}}$ is calculated, as shown in Figure 8 .

Similar with the inner-ring vertical dynamic response displayed in Figure 6, the vertical vibration of the outer ring exhibits obvious periodicity, and the overall amplitude increases with temperature. The frequency domain result is shown in Figure 9.

Compared with the inner ring velocities, the frequency domain results for outer ring velocities contain more peak frequencies. The peak frequencies at $40 \mathrm{~Hz}, 80 \mathrm{~Hz}$, and $160 \mathrm{~Hz}$ are 1,2 , and 4 times rotating frequencies, respectively. The 3 times rotating frequency is close to the defect frequency, and the amplitudes at 3 times the rotating frequency are, therefore, hard to distinguish. The defect 


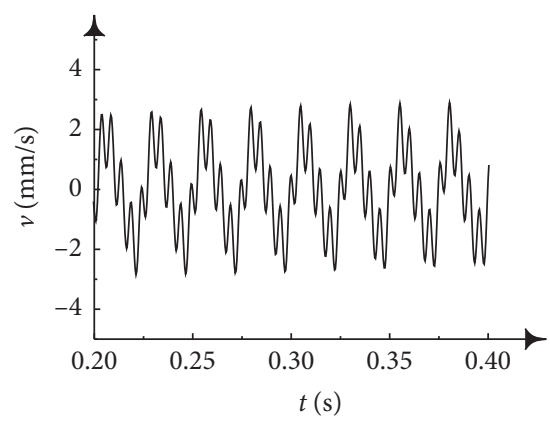

(a)

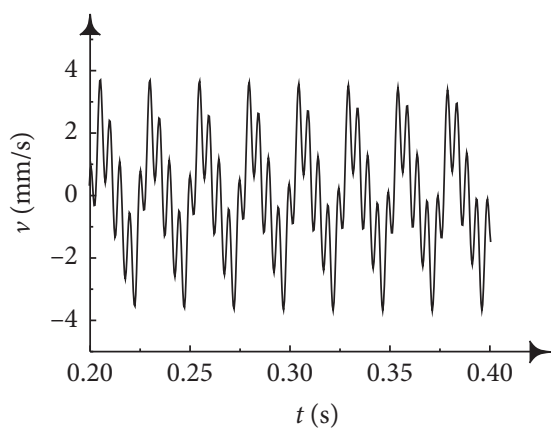

(d)

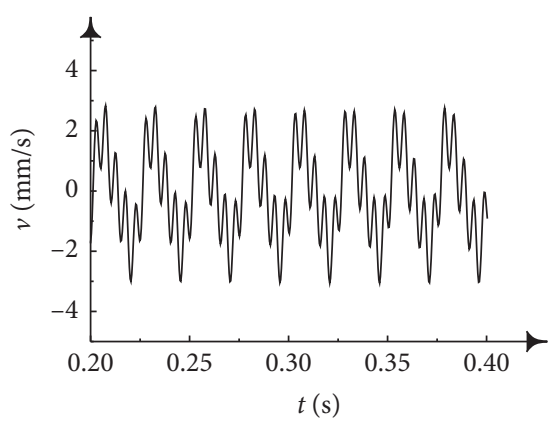

(b)

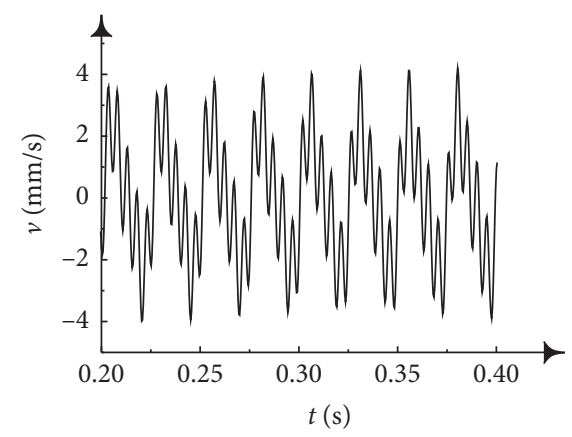

(e)

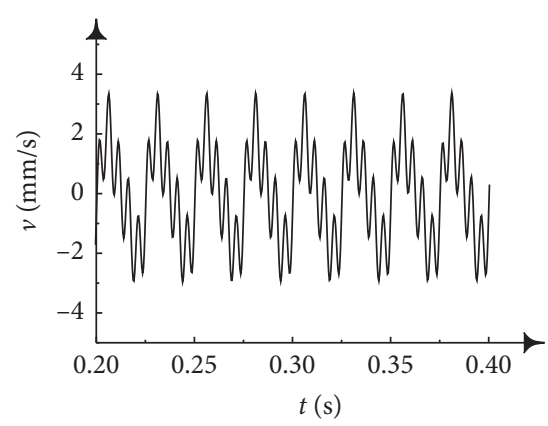

(c)

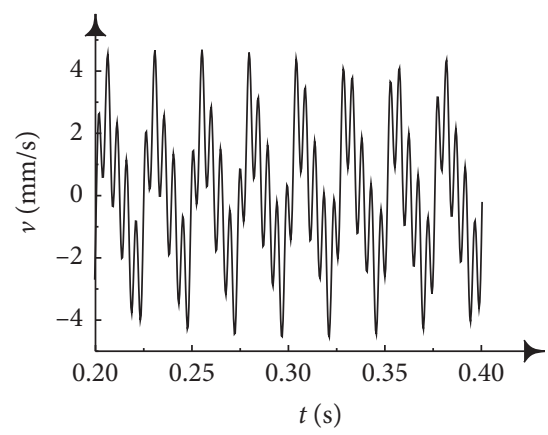

(f)

Figure 6: Vertical velocities of the inner ring in various temperatures. (a) $T=100 \mathrm{~K}$. (b) $T=200 \mathrm{~K}$. (c) $T=300 \mathrm{~K}$. (d) $T=400 \mathrm{~K}$. (e) $T=500 \mathrm{~K}$. (f) $T=600 \mathrm{~K}$.

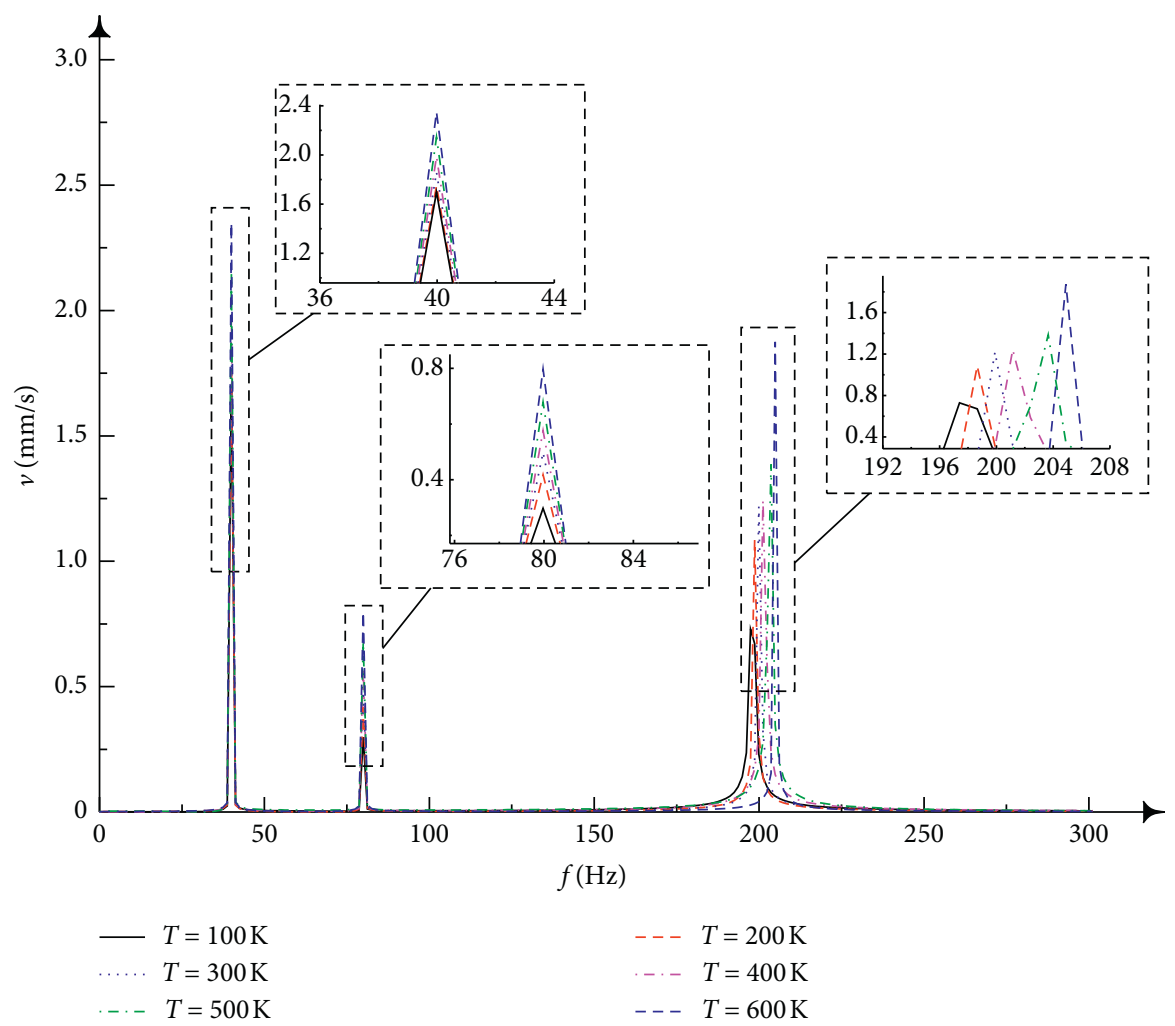

FIGURE 7: Frequency domain results for inner ring velocities. 
TABLE 3: Peak frequencies and amplitudes of inner ring vibration in different temperatures.

\begin{tabular}{|c|c|c|c|c|c|c|}
\hline Temperature $(\mathrm{K})$ & 100 & 200 & 300 & 400 & 500 & 600 \\
\hline Amplitude at the rotating frequency $(\mathrm{mm} / \mathrm{s})$ & 1.70 & 1.75 & 1.86 & 1.99 & 2.14 & 2.35 \\
\hline Amplitude at the doubling rotating frequency $(\mathrm{mm} / \mathrm{s})$ & 0.30 & 0.42 & 0.50 & 0.58 & 0.68 & 0.80 \\
\hline Defect frequency $(\mathrm{Hz})$ & 197.40 & 198.65 & 199.90 & 201.15 & 203.65 & 204.90 \\
\hline Amplitude at the defect frequency $(\mathrm{mm} / \mathrm{s})$ & 0.72 & 1.08 & 1.21 & 1.24 & 1.39 & 1.87 \\
\hline
\end{tabular}

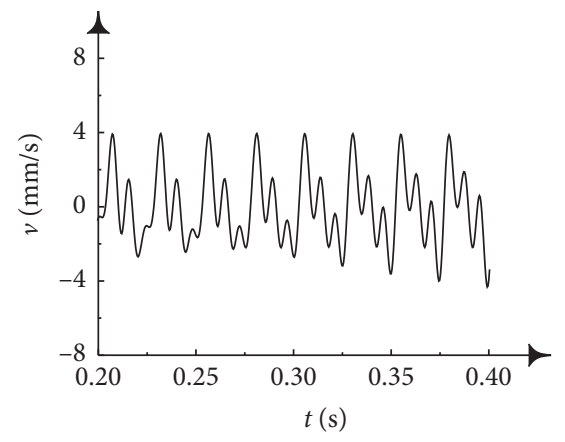

(a)

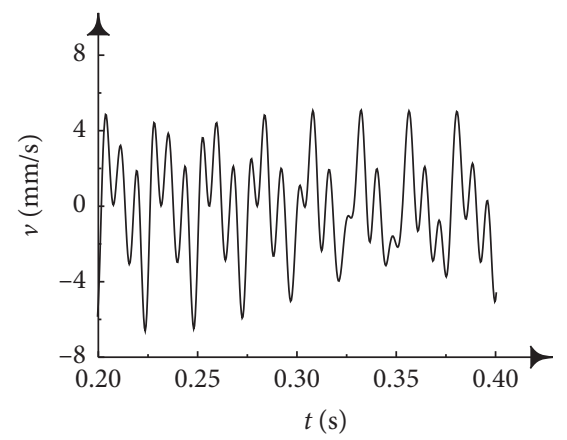

(d)

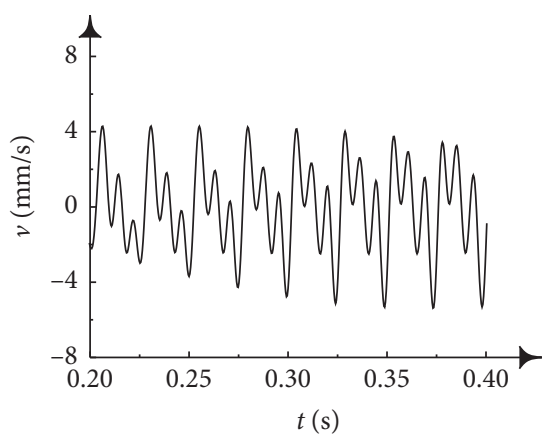

(b)

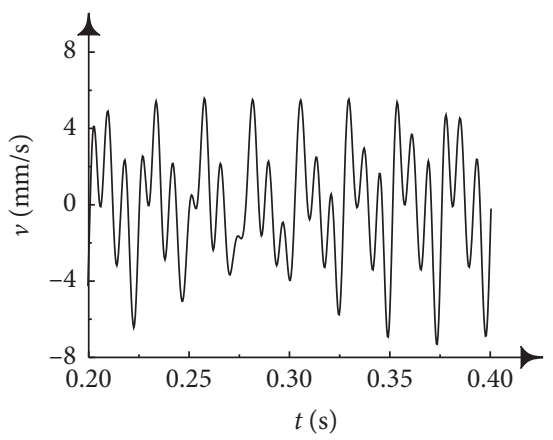

(e)

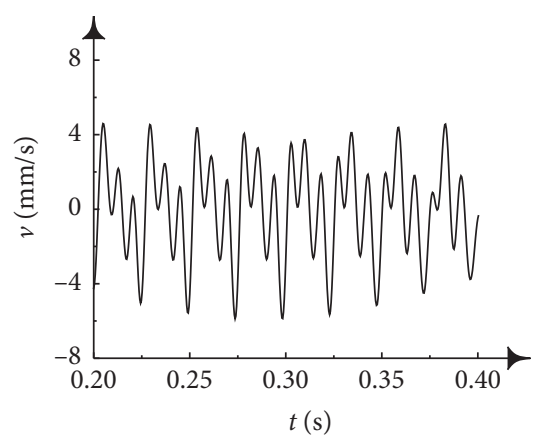

(c)

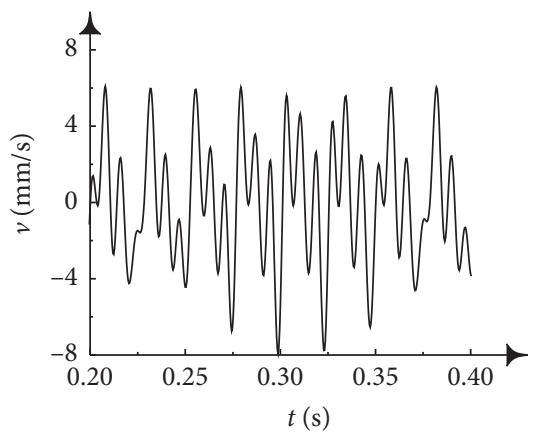

(f)

Figure 8: Vertical velocities of the outer ring in various temperatures. (a) $T=100 \mathrm{~K}$. (b) $T=200 \mathrm{~K}$. (c) $T=300 \mathrm{~K}$. (d) $T=400 \mathrm{~K}$. (e) $T=500 \mathrm{~K}$. (f) $T=600 \mathrm{~K}$.

frequency and amplitudes at each peak in different temperatures are given in Table 4.

As shown in Figure 9 and Table 5, the amplitudes at the characteristic frequencies related with the rotating speed generally increase with temperature, indicating that the centrifugal force, which increases with the enlarged fit clearance, is the main factor affecting the responses at these frequencies. When the outer ring makes a spinning motion reverse to the inner ring in the fit clearance, the orbit speed of the balls decreases, and the impact frequency caused by the outer ring defect increases according to equation (19). Therefore, the defect frequency grows with temperature, and the amplitude of the defect frequency also increases with the centrifugal force basically.

\section{Experimental Investigation}

To test the accuracy of the dynamic model with raceway defects at different temperatures, an experimental investigation is conducted on a rotor-bearing test rig to compare with the calculation results. The test rig is displayed in Figure 10.
In Figure 10, the ceramic bearings are installed in the steel pedestals, and a pedestal is placed in an incubator. The structural parameters of the bearing in the experiment are the same as in Table 1. The shaft is driven by the motor through the coupling, and the inner rings of the bearings run synchronously with the shaft. The rotation speed of the shaft can be adjusted manually through the motor frequency, and the plates on the shaft act as the radial load. The incubator is set for heat preservation and covers a bearing and a pedestal. The remote sensing thermometer is used to obtain the temperature, and the accelerometer is set to get the dynamic response. At the beginning, the liquid nitrogen is poured into the incubator to lower the working temperature. The rotation speed of the motor is set stable as $2400 \mathrm{r} \mathrm{min}^{-1}$; the weight of the rotor and plates is $100 \mathrm{~N}$. Then, the working temperature gradually increases due to the heat generation and exchange, and the wide temperature ranges are simulated in the incubator. Enough liquid nitrogen can keep the initial temperature below $100 \mathrm{~K}$, and the laboratory temperature is $298 \mathrm{~K}$. After reaching the laboratory temperature, the working temperature continues to rise due to the heat production of the bearing and finally stops at about $340 \mathrm{~K}$. To 


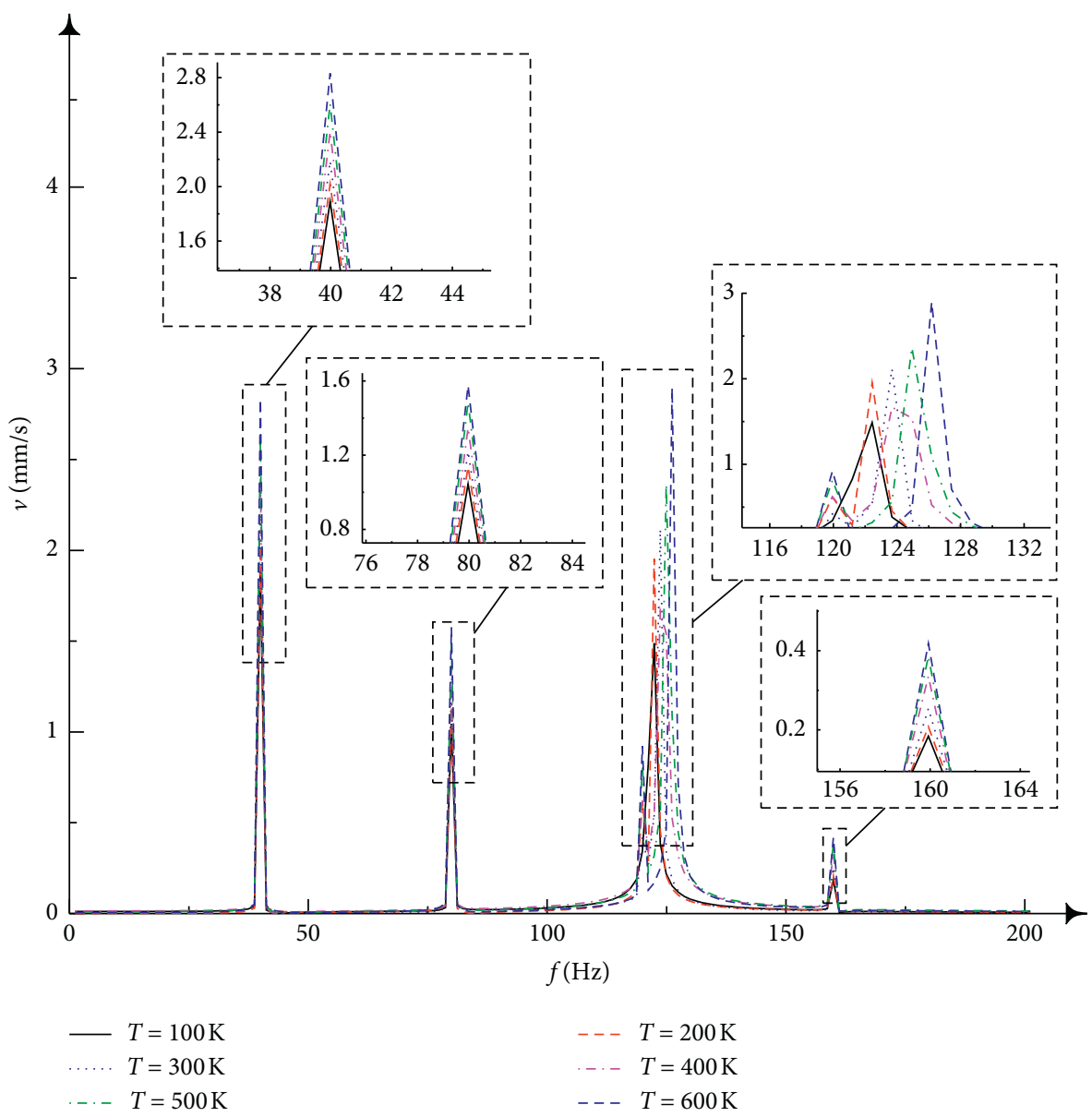

Figure 9: Frequency domain results for outer ring velocities.

TABLE 4: Peak frequencies and amplitudes of the outer ring vibration in different temperatures.

\begin{tabular}{|c|c|c|c|c|c|c|}
\hline Temperature $(\mathrm{K})$ & 100 & 200 & 300 & 400 & 500 & 600 \\
\hline Amplitude at the $1 \times$ rotating frequency $(\mathrm{mm} / \mathrm{s})$ & 1.89 & 2.02 & 2.19 & 2.40 & 2.61 & 2.83 \\
\hline Amplitude at the $2 \times$ rotating frequency $(\mathrm{mm} / \mathrm{s})$ & 1.04 & 1.14 & 1.22 & 1.35 & 1.48 & 1.58 \\
\hline Amplitude at the $3 \times$ rotating frequency $(\mathrm{mm} / \mathrm{s})$ & 0.34 & 0.62 & 0.42 & 0.62 & 0.81 & 0.92 \\
\hline Amplitude at the $4 \times$ rotating frequency $(\mathrm{mm} / \mathrm{s})$ & 0.18 & 0.21 & 0.25 & 0.34 & 0.39 & 0.42 \\
\hline Defect frequency $(\mathrm{Hz})$ & 122.44 & 122.44 & 123.69 & 123.69 & 124.94 & 126.19 \\
\hline Amplitude at the defect frequency $(\mathrm{mm} / \mathrm{s})$ & 1.49 & 1.95 & 2.11 & 1.67 & 2.36 & 2.89 \\
\hline
\end{tabular}

TABle 5: Comparison between the simulation and experiment results.

\begin{tabular}{lcccc}
\hline $\begin{array}{l}\text { Temperature } \\
(\mathrm{K})\end{array}$ & $\begin{array}{c}\text { Simulation result of inner } \\
\text { raceway defect frequency } \\
(\mathrm{Hz})\end{array}$ & $\begin{array}{c}\text { Experimental result of inner } \\
\text { raceway defect frequency }(\mathrm{Hz})\end{array}$ & $\begin{array}{c}\text { Simulation result of outer } \\
\text { raceway defect frequency } \\
(\mathrm{Hz})\end{array}$ & $\begin{array}{c}\text { Experimental result of outer } \\
\text { raceway defect frequency }(\mathrm{Hz})\end{array}$ \\
\hline 100 & 197.40 & 197.80 & 122.43 & 122.80 \\
200 & 198.65 & 199.00 & 122.43 & 123.20 \\
300 & 199.90 & 200.40 & 123.69 & 124.00 \\
\hline
\end{tabular}

get the performance of the bearing with temperature ranges, the signals at $T=100 \mathrm{~K}, 200 \mathrm{~K}$, and $300 \mathrm{~K}$ are collected. The bearing near the coupling is a healthy bearing, while the bearing in the incubator is with a raceway defect. The defects on the inner and outer raceways are initially located at the bottom positions, with $\psi_{s i}=\phi_{s o}=270^{\circ}$. The sampling frequency is set as $16384 \mathrm{~Hz}$. Two cases with the inner ring defect and outer ring defect are investigated in the experiment, and the vertical accelerations at the pedestal are collected, as shown in Figure 11.

Two cases with the inner raceway defect and outer raceway defect are shown in Figure 11. The black solid lines show the performance at $T=100 \mathrm{~K}$, while the red dashed lines and blue dot-dashed lines show the performances at 

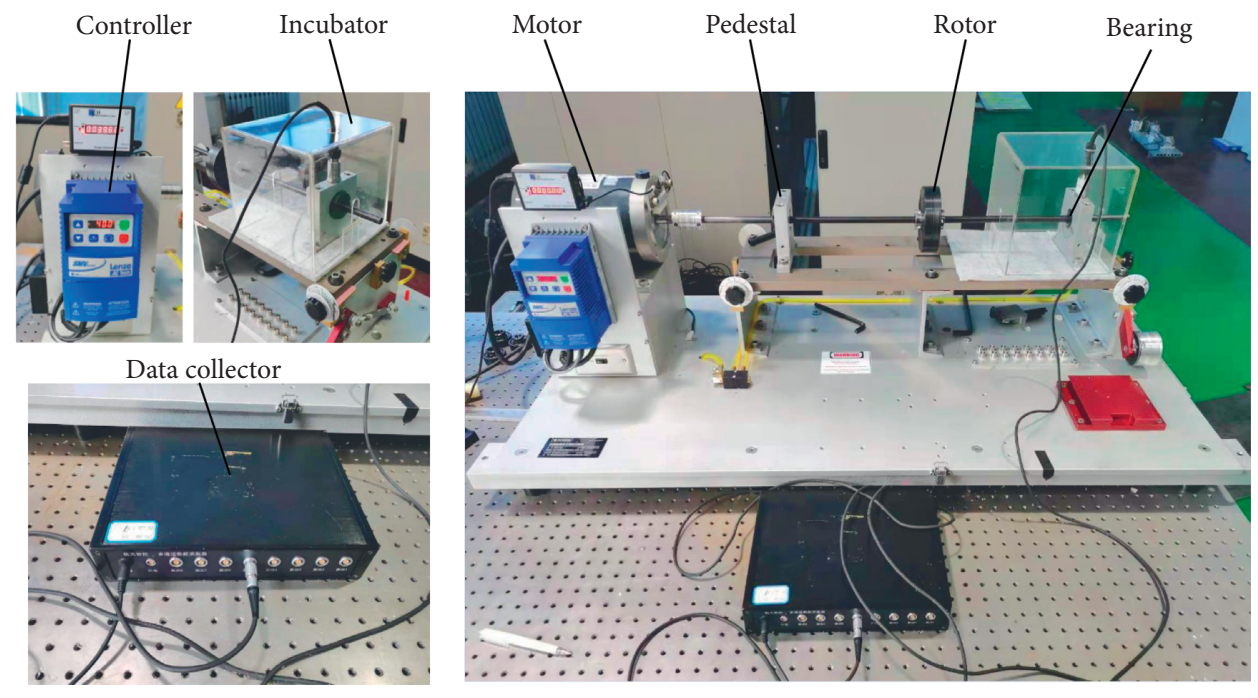

Figure 10: Test rig used in the experiment.

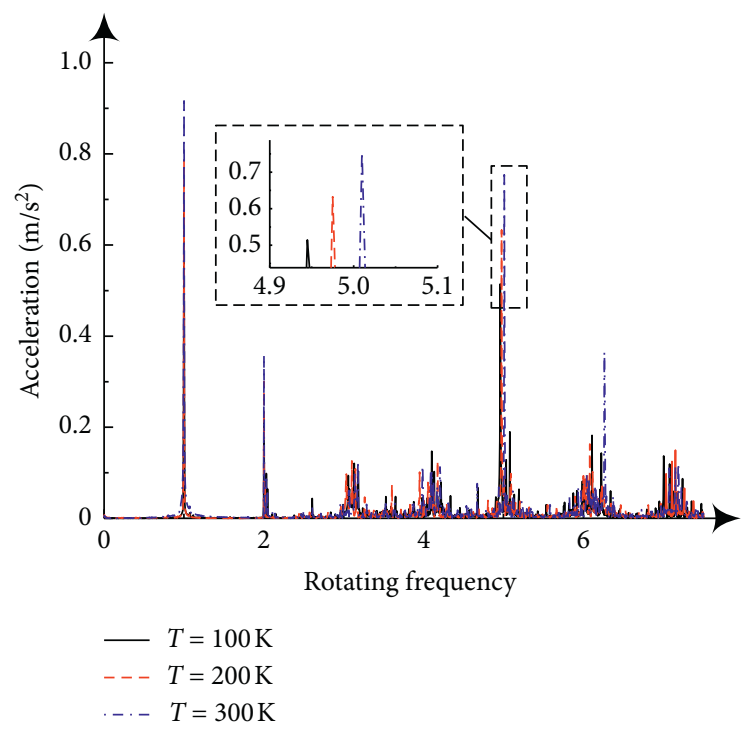

(a)

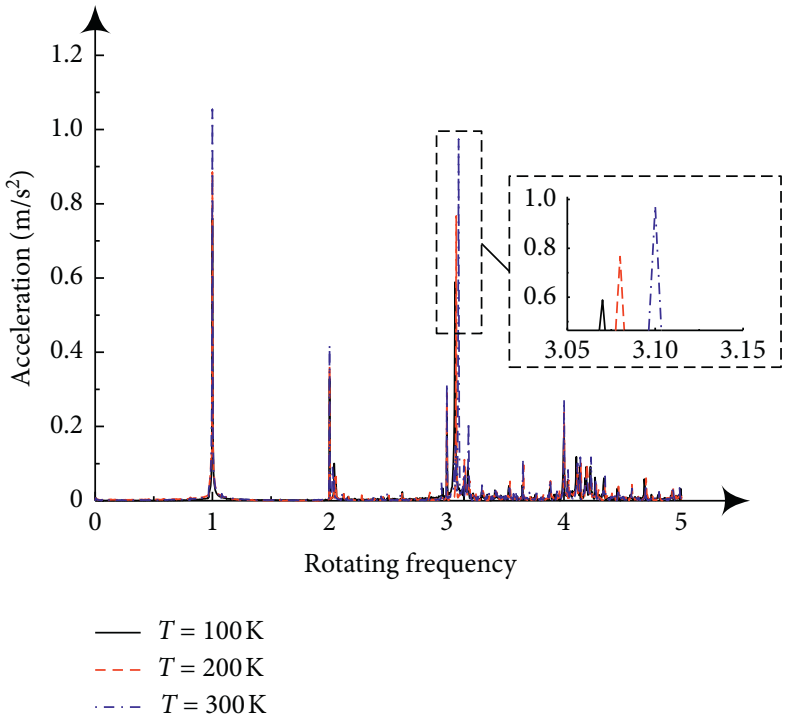

(b)

Figure 11: Vertical accelerations at different temperatures with different defects. (a) Inner raceway defect. (b) Outer raceway defect.

$T=200 \mathrm{~K}$ and $300 \mathrm{~K}$, respectively. In Figure 11(a), the peaks at $1 \times$ and $2 \times$ rotating frequencies are in consistence in different temperatures. However, for the defect frequency, which occurs at about the $5 \times$ rotating frequency, the peak deviates with temperature. It can be seen that the peak frequency for the inner raceway defect increases with working temperature, and the amplitude also grows. Similar situations can also be seen in Figure 11(b), the peaks at $1 \times, 2 \times, 3 \times$, and $4 \times$ rotating frequencies remain unchanged in the wide temperature range, but the characteristic frequency for the outer raceway defect moves towards right. The amplitudes at the $1 \times$ rotating frequency and defect frequency increase significantly and vary less at higher orders of rotating frequencies, and the trends are in consistent with the simulation results. The comparison between the defect frequencies in simulation and experimental results is shown in Table 5.

As shown in Table 5, the defect frequencies of the experimental results match well with the simulation results and have the same trends with working temperature. Therefore, it can be inferred that the dynamic model with temperaturerelated fit clearance is suitable for the analysis of the fullceramic bearing system, and the defect frequencies deviate with the working temperature.

\section{Discussion}

As shown in the simulation and experimental results, when the working temperature of the full-ceramic bearing system increases, the fit clearance between the outer ring 
and the pedestal increases. Due to the friction forces from the balls and pedestal, the outer ring spins in the reverse direction to the inner ring, leading to the increase of inner raceway defect and outer raceway defect characteristic frequencies. On the contrary, the motion of the outer ring in the fit clearance makes the dynamic response of the bearing system more complex and brings about more frequency components related with the rotation speed. The outer ring is more affected by the friction and impact forces from the pedestal; therefore, compared with the dynamic response with the inner raceway defect, the dynamic response with the outer raceway defect contains more frequency components related with the rotation speed. When the working temperature is fixed, the rotation speed of the outer ring can be regarded as a constant value, and the rotation speed affects the defect frequency proportionally. As a result, the characteristic frequency of the outer raceway defect has less deviation with temperature compared with that of the inner raceway defect. In Table 4, the defect frequency has no change from $T=100 \mathrm{~K}$ to $200 \mathrm{~K}$ and $300 \mathrm{~K}$ to $400 \mathrm{~K}$. This is because the sampling frequency is not high enough, and the frequency deviation is smaller than the frequency step. According to the simulation results, the inner raceway defect frequency has a deviation of $3.1 \%$ and the outer raceway defect frequency has a deviation of $3.8 \%$ in the wide temperature range from $100 \mathrm{~K}$ to $600 \mathrm{~K}$. Therefore, it can be inferred that the deviation should be considered to make it more accurate for the status monitoring of the full-ceramic bearing system.

\section{Conclusions}

The dynamic model of full-ceramic ball bearing systems with the effect of temperature-related fit clearance is established in this paper, and the motions of the bearing rings are calculated at different temperatures. The defect frequencies are investigated, and the simulation results are verified through experiments. Results show that when the temperature increases, the fit clearance grows due to the thermal deformation difference. The peak frequencies related with the rotation speed stay still, and the amplitudes grow due to the centrifugal force. The outer ring spins in the reverse direction to the inner ring, thus changing the relative motion between balls and rings and leading to the deviation of defect frequencies. The spinning speed of the outer ring increases with the temperature, and the characteristic frequencies for the inner raceway and outer raceway grow monotonously. The defect frequencies change in proportion, and the deviation of inner raceway defect frequency is smaller than that of outer raceway defect frequency. The defect frequencies at different temperatures can be revised through the results in this study, and the research findings provide theoretical foundations for fault diagnosis in wide temperature ranges. The results in this study also proved that the defect frequency deviation needs to be considered in the condition monitoring of full-ceramic bearing systems, and the defect frequencies need to be revised.

\section{Data Availability}

The data used to support the findings of this study are included within this article.

\section{Conflicts of Interest}

The authors declare that there are no conflicts of interest regarding the publication of this paper.

\section{Acknowledgments}

This research was funded by National Science Foundation of China (Grant nos. 51905357 and 52075348) and Education Department Project of Liaoning Province (Grant no. lnqn201909).

\section{References}

[1] H. T. Shi and X. T. Bai, "Model-based uneven loading condition monitoring of full ceramic ball bearings in starved lubrication," Mechanical Systems and Signal Processing, vol. 139, Article ID 106583, 2020.

[2] H. T. Shi, X. T. Bai, and K. Zhang, "Effect of thermal-related fit clearance between outer ring and pedestal on the vibration of full ceramic ball bearing," Shock and Vibration, vol. 2019, Article ID 8357807, , 2019.

[3] V. Parmar, V. H. Saran, and S. P. Harsha, "Effect of an unbalanced rotor on dynamic characteristics of double-row selfaligning ball bearing," European Journal of Mechanics - A/ Solids, vol. 82, Article ID 104006, 2020.

[4] K. Yu, T. R. Lin, and H. Ma, "A multi-stage semi-supervised learning approach for intelligent fault diagnosis of rolling bearing using data augmentation and metric learning," $M e$ chanical Systems and Signal Processing, vol. 146, Article ID 107043, 2021.

[5] Y. Qin, C. C. Li, and X. G. Wu, "Multiple-degree-of-freedom dynamic model of rolling bearing with a localized surface defect," Mechanism and Machine Theory, vol. 154, Article ID 104047, 2020.

[6] M. X. Hou and H. T. Shi, "Stator-winding incipient shortedturn fault detection for motor system in motorized spindle using modified interval observers," IEEE Transactions on Instrumentation and Measurement, vol. 70, 2020.

[7] K. Yu, Q. Fu, H. Ma, T. R. Lin, and X. Li, "Simulation data driven weakly supervised adversarial domain adaptation approach for intelligent cross-machine fault diagnosis," Structural Health Monitoring, vol. 718, 2021.

[8] S. Xi, H. Cao, and X. Chen, "Dynamic modeling of spindle bearing system and vibration response investigation," $\mathrm{Me}$ chanical Systems and Signal Processing, vol. 114, pp. 486-511, 2019.

[9] L. Bizarre, F. Nonato, and K. L. Cavalca, "Formulation of five degrees of freedom ball bearing model accounting for the nonlinear stiffness and damping of elastohydrodynamic point contacts," Mechanism and Machine Theory, vol. 124, pp. 179-196, 2018.

[10] X. Li, K. Yu, and H. Ma, “Analysis of varying contact angles and load distributions in defective angular contact ball bearing," Engineering and Failure Analysis, vol. 91, pp. 449464, 2019.

[11] C. Yan, M. Zhao, and J. Lin, "Fault signature enhancement and skidding evaluation of rolling bearing based on estimating 
the phase of the impulse envelope signal," Journal of Sound and Vibration, vol. 485, Article ID 115529, 2020.

[12] J. C. C. Molano, L. Capelli, and R. Rubini, "A bearing fault model for Independent Cart Conveyor System and its validation," Applied Acoustics, vol. 159, Article ID 107069, 2020.

[13] H. Yu, Y. Ran, G. B. Zhang, X. L. Li, and B. R. Li, “A bearing fault model for Independent Cart Conveyor System and its validation," Journal of Sound and Vibration, vol. 488, Article ID 115650, 2020.

[14] M. Lal, "Modeling and estimation of speed dependent bearing and coupling misalignment faults in a turbine generator system," Mechanical Systems and Signal Processing, vol. 151, Article ID 107365, 2021.

[15] A. Kumar, C. P. Gandhi, and Y. Q. Zhou, "Variational mode decomposition based symmetric single valued neutrosophic cross entropy measure for the identification of bearing defects in a centrifugal pump," Applied Acoustics, vol. 165, Article ID 107294, 2020.

[16] L. Zhang, H. Xu, and S. L. Zhang, "A radial clearance adjustable bearing reduces the vibration response of the rotor system during acceleration," Tribology International, vol. 144, Article ID 106112, 2020.

[17] L. B. Visnadi and H. F. de Castro, "Influence of bearing clearance and oil temperature uncertainties on the stability threshold of cylindrical journal bearings," Mechanism and Machine Theory, vol. 134, pp. 57-73, 2019.

[18] G. Chen and M. Qu, "Modeling and analysis of fit clearance between rolling bearing outer ring and housing," Journal of Sound and Vibration, vol. 438, pp. 419-440, 2019.

[19] H. R. Cao, F. Shi, and Y. M. Li, "Vibration and stability analysis of rotor-bearing-pedestal system due to clearance fit," Mechanical Systems and Signal Processing, vol. 133, Article ID 106275, 2019.

[20] Y. Mao, L. Wang, and C. Zhang, "Influence of ring deformation on the dynamic characteristics of a roller bearing in clearance fit with housing," International Journal of $\mathrm{Me}$ chanical Sciences, vol. 139, pp. 122-130, 2018.

[21] H. T. Shi, X. T. Bai, and K. Zhang, "Influence of uneven loading condition on the sound radiation of starved lubricated full ceramic ball bearings," Journal of Sound and Vibration, vol. 461, Article ID 114910, 2019.

[22] H. T. Shi, Y. Y. Li, and X. T. Bai, "Investigation of the orbitspinning behaviors of the outer ring in a full ceramic ball bearing-steel pedestal system in wide temperature ranges," Mechanical Systems and Signal Processing, vol. 149, Article ID 107317, 2021. 